\title{
XOR gate response in a mesoscopic ring with embedded quantum dots
}

\author{
Santanu K. Maiti ${ }^{1,2, *}$ \\ ${ }^{1}$ Theoretical Condensed Matter Physics Division, Saha Institute of Nuclear Physics, \\ 1/AF, Bidhannagar, Kolkata-700 064, India \\ ${ }^{2}$ Department of Physics, Narasinha Dutt College, 129, Belilious Road, Howrah-711 101, India
}

\begin{abstract}
We address XOR gate response in a mesoscopic ring threaded by a magnetic flux $\phi$. The ring, composed of identical quantum dots, is symmetrically attached to two semi-infinite one-dimensional metallic electrodes and two gate voltages, viz, $V_{a}$ and $V_{b}$, are applied, respectively, in each arm of the ring which are treated as the two inputs of the XOR gate. The calculations are based on the tight-binding model and the Green's function method, which numerically compute the conductance-energy and current-voltage characteristics as functions of the ring-electrodes coupling strengths, magnetic flux and gate voltages. Quite interestingly it is observed that, for $\phi=\phi_{0} / 2$ ( $\phi_{0}=c h / e$, the elementary flux-quantum) a high output current (1) (in the logical sense) appears if one, and only one, of the inputs to the gate is high (1), while if both inputs are low (0) or both are high (1), a low output current (0) appears. It clearly demonstrates the XOR behavior and this aspect may be utilized in designing the electronic logic gate.
\end{abstract}

PACS No.: 73.23.-b; 73.63.Rt.

Keywords: A. Mesoscopic ring; D. Conductance; D. I-V characteristic; D. XOR gate.

*Corresponding Author: Santanu K. Maiti

Electronic mail: santanu.maiti@saha.ac.in 


\section{Introduction}

In the present age of nanoscience and technology quantum confined model systems are used extensively in electronic as well as spintronic engineering since these simple looking systems are the fundamental building blocks of designing nano devices. A mesoscopic normal metal ring is one such promising example of quantum confined systems. Here we will explore the electron transport through a mesoscopic ring, composed of identical quantum dots and attached to two external electrodes, the so-called electrode-ring-electrode bridge, and show how such a simple geometric model can be used to design a logic gate. The theoretical description of electron transport in a bridge system has been followed based on the pioneering work of Aviram and Ratner 11. Later, many excellent experiments [2, 3, 4] have been done in several bridge systems to understand the basic mechanisms underlying the electron transport. Though in literature many theoretical [5, 6, 7, 8, 9, 10, 11, 12, 13, 14, as well as experimental papers [2, 3, 4, on electron transport are available, yet lot of controversies are still present between the theory and experiment, and the complete knowledge of the conduction mechanism in this scale is not very well established even today. The electronic transport in the ring significantly depends on the ring-to-electrodes interface structure. By changing the geometry one can tune the transmission probability of an electron across the ring. This is solely due to the quantum interference effect among the electronic waves traversing through different arms of the ring. Furthermore, the electron transport through the ring can be modulated in other way by tuning the magnetic flux, the socalled Aharonov-Bohm (AB) flux, that threads the ring. The $\mathrm{AB}$ flux threading the ring can change the phases of the wave functions propagating along the different arms of the ring leading to constructive or destructive interferences, and accordingly the transmission amplitude changes [15, 16, 17, 18, 19. Beside these factors, ring-to-electrodes coupling is another important issue that controls the electron transport in a meaningful way [19. All these are the key factors which regulate the electron transmission in the electrode-ring-electrode bridge system and these effects have to be taken into account properly to reveal the transport mechanisms.

The aim of the present paper is to describe the XOR gate response in a mesoscopic ring threaded by a magnetic flux $\phi$. The ring is contacted symmetrically to the electrodes, and the two arms of the ring are subjected to two gate voltages $V_{a}$ and $V_{b}$, respectively (see Fig. 1) those are treated as the two inputs of the XOR gate. Here we adopt a simple tight-binding model to describe the system and all the calculations are performed numerically. We address the XOR behavior by studying the conductance-energy and current-voltage characteristics as functions of the ring-electrodes coupling strengths, magnetic flux and gate voltages. Our study reveals that for a particular value of the magnetic flux, $\phi=\phi_{0} / 2$, a high output current (1) (in the logical sense) is available if one, and only one, of the inputs to the gate is high (1), while if both the inputs are low (0) or both are high (1), a low output current (0) is available. This phenomenon clearly demonstrates the XOR behavior which may be utilized in manufacturing the electronic logic gate. To the best of our knowledge the XOR gate response in such a simple system has not been described earlier in the literature.

The paper is organized as follow. Following the introduction (Section 1), in Section 2, we present the model and the theoretical formulations for our calculations. Section 3 discusses the significant results, and finally, we summarize our results in Section 4.

\section{Model and the synopsis of the theoretical background}

We begin by referring to Fig. 1 A mesoscopic ring, composed of identical quantum dots (filled red circles) and threaded by a magnetic flux $\phi$, is attached symmetrically to two semi-infinite one-dimensional metallic electrodes. The ring is placed between two gate electrodes, viz, gate-a and gate-b. These gate electrodes are ideally isolated from the ring and can be regarded as two parallel plates of a capacitor. In our present scheme we assume that the gate voltages each operate on the dots nearest to the plates only. While, in complicated geometric models, the effect must be taken into account for the other dots, though the effect becomes too small. The dots $a$ and $b$ in the two arms of the ring are subjected to the gate voltages $V_{a}$ and $V_{b}$, respectively, and these are treated as the two inputs of the XOR gate. The actual scheme of connections with the batteries for the operation of the XOR gate is clearly presented in the figure (Fig. 10), where the source and the gate voltages are applied with respect to the drain.

Based on the Landauer conductance formula 20 , 21] we determine the conductance $(g)$ of the ring. 
At very low temperature and bias voltage it can be expressed in the form,

$$
g=\frac{2 e^{2}}{h} T
$$

where $T$ gives the transmission probability of an electron through the ring. This $(T)$ can be represented in terms of the Green's function of the ring and its coupling to the two electrodes by the relation [20, 21],

$$
T=\operatorname{Tr}\left[\Gamma_{S} G_{R}^{r} \Gamma_{D} G_{R}^{a}\right]
$$

where $G_{R}^{r}$ and $G_{R}^{a}$ are respectively the retarded and advanced Green's functions of the ring including the effects of the electrodes. The parameters $\Gamma_{S}$ and $\Gamma_{D}$ describe the coupling of the ring to the source and

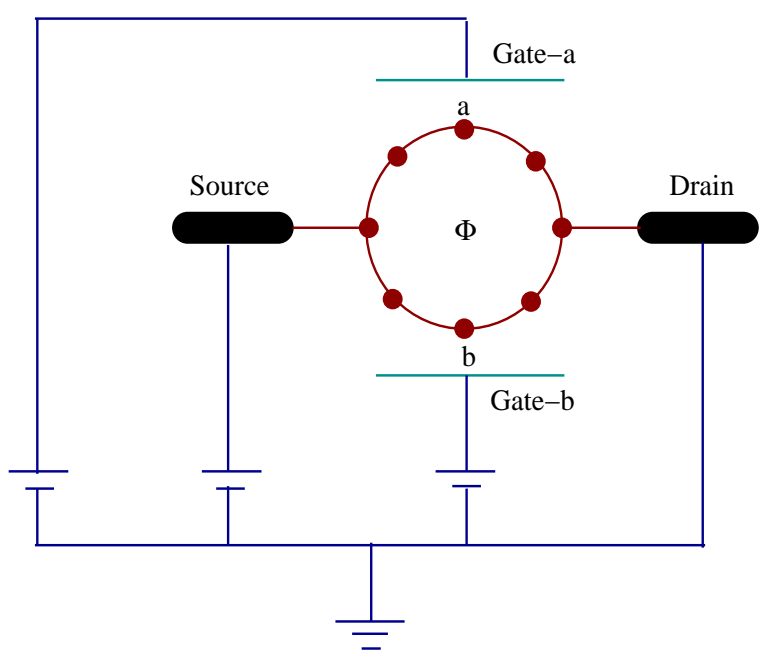

Figure 1: (Color online). The scheme of connections with the batteries for the operation of the XOR gate. A mesoscopic ring with embedded quantum dots (filled red circles) is attached to two semi-infinite one-dimensional metallic electrodes, viz, source and drain. The gate voltages $V_{a}$ and $V_{b}$, those are variable, are applied in the dots $a$ and $b$ of the two arms, respectively. The source and the gate voltages are applied with respect to the drain.

drain, respectively. For the full system i.e., the ring, source and drain, the Green's function is defined as,

$$
G=(E-H)^{-1}
$$

where $E$ is the injecting energy of the source electron. To Evaluate this Green's function, the inversion of an infinite matrix is needed since the full system consists of the finite ring and the two semiinfinite electrodes. However, the entire system can be partitioned into sub-matrices corresponding to the individual sub-systems and the Green's function for the ring can be effectively written as,

$$
G_{R}=\left(E-H_{R}-\Sigma_{S}-\Sigma_{D}\right)^{-1}
$$

where $H_{R}$ is the Hamiltonian of the ring that can be expressed within the non-interacting picture like,

$$
\begin{aligned}
H_{R} & =\sum_{i}\left(\epsilon_{i 0}+V_{a} \delta_{i a}+V_{b} \delta_{i b}\right) c_{i}^{\dagger} c_{i} \\
& +\sum_{<i j>} t\left(c_{i}^{\dagger} c_{j} e^{i \theta}+c_{j}^{\dagger} c_{i} e^{-i \theta}\right)
\end{aligned}
$$

In this Hamiltonian $\epsilon_{i 0}$ 's are the on-site energies for all the sites (filled red circles) $i$, except the sites $i=a$ and $b$ where the gate voltages $V_{a}$ and $V_{b}$ are applied, those are variable. These gate voltages can be incorporated through the site energies as expressed in the above Hamiltonian. $c_{i}^{\dagger}\left(c_{i}\right)$ is the creation (annihilation) operator of an electron at the site $i$ and $t$ is the nearest-neighbor hopping integral. $\theta=2 \pi \phi / N \phi_{0}$ is the phase factor due to the flux $\phi$, where $N$ represents the total number of sites/dots in the ring. Similar kind of tight-binding Hamiltonian is also used, except the phase factor $\theta$, to describe the semi-infinite one-dimensional perfect electrodes where the Hamiltonian is parametrized by constant on-site potential $\epsilon_{0}$ and nearest-neighbor hopping integral $t_{0}$. The ring is coupled to the electrodes by the parameters $\tau_{S}$ and $\tau_{D}$, where they (coupling parameters) correspond to the coupling strengths with the source and drain, respectively. The parameters $\Sigma_{S}$ and $\Sigma_{D}$ in Eq. (4) represent the self-energies due to the coupling of the ring to the source and drain, respectively, where all the informations of this coupling are included into these self-energies.

To evaluate the current $(I)$, passing through the ring, as a function of the applied bias voltage $(V)$ we use the relation [20,

$$
I(V)=\frac{e}{\pi \hbar} \int_{E_{F}-e V / 2}^{E_{F}+e V / 2} T(E) d E
$$

where $E_{F}$ is the equilibrium Fermi energy. Here we make a realistic assumption that the entire voltage is dropped across the ring-electrode interfaces, and it is examined that under such an assumption the $I-V$ characteristics do not change their qualitative features.

In this presentation, all the results are computed only at absolute zero temperature. These results 
are also valid even for some finite (low) temperatures, since the broadening of the energy levels of the ring due to its coupling with the electrodes becomes much larger than that of the thermal broadening 20. On the other hand, at high temperature limit, all these phenomena completely disappear. This is due to the fact that the phase coherence length decreases significantly with the rise of temperature where the contribution comes mainly from the scattering on phonons, and accordingly, the quantum interference effect vanishes. For the sake of simplicity, we take the unit $c=e=h=1$ in our present calculations.

\section{Results and discussion}

To illustrate the results, let us first mention the values of the different parameters used for the numerical calculations. In the ring, the on-site energy $\epsilon_{i 0}$ is taken as 0 for all the sites $i$, except the sites $i=a$ and $b$ where the site energies are taken as $V_{a}$ and $V_{b}$, respectively, and the nearest-neighbor
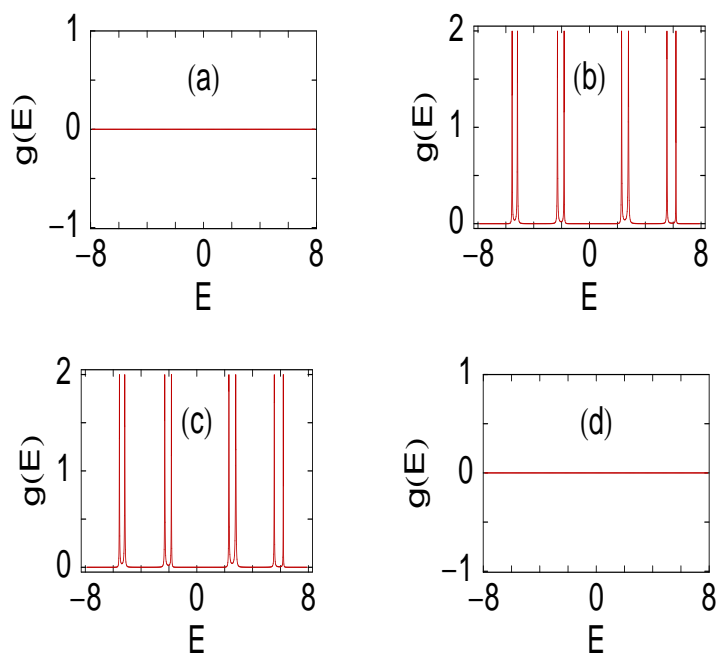

Figure 2: (Color online). Conductance $g$ as a function of the energy $E$ for a mesoscopic ring with $N=8$ and $\phi=0.5$ in the limit of weak-coupling. (a) $V_{a}=V_{b}=0$, (b) $V_{a}=2$ and $V_{b}=0$, (c) $V_{a}=0$ and $V_{b}=2$ and (d) $V_{a}=V_{b}=2$.

hopping strength $t$ is set to 3 . On the other hand, for the side attached electrodes the on-site energy $\left(\epsilon_{0}\right)$ and the nearest-neighbor hopping strength $\left(t_{0}\right)$ are fixed to 0 and 4, respectively. The Fermi energy $E_{F}$ is set to 0 . To narrate the coupling effect, throughout the study we focus our results for the two limiting cases depending on the strength of the coupling of the ring to the source and drain. Case $I$ : The weak-coupling limit. It is described by the condition $\tau_{S(D)}<<t$. For this regime we choose $\tau_{S}=\tau_{D}=0.5$. Case $I I$ : The strong-coupling limit. This is specified by the condition $\tau_{S(D)} \sim t$. In this particular regime, we set the values of the parameters as $\tau_{S}=\tau_{D}=2.5$. The key controlling parameter for all these calculations is the magnetic flux $\phi$ which is set to $\phi_{0} / 2$ i.e., 0.5 in our chosen unit $c=e=h=1$.

In Fig. 2 we present the conductance-energy ( $g$ $E$ ) characteristics for a mesoscopic ring with $N=8$ in the limit of weak-coupling, where (a), (b), (c) and (d) correspond to the results for the different gate voltages. When both the two inputs $V_{a}$ and $V_{b}$ are identical to zero i.e., both the inputs are low, the conductance $g$ becomes exactly zero (Fig. 2(a))
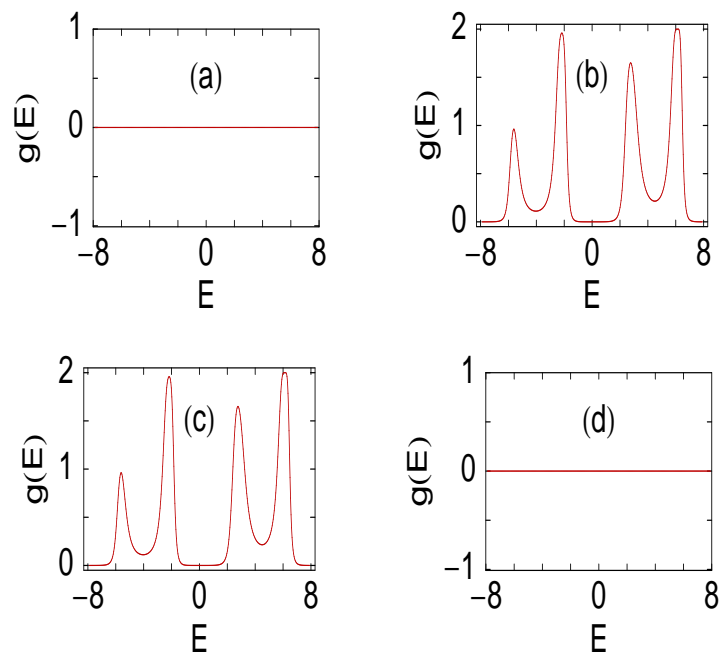

Figure 3: (Color online). Conductance $g$ as a function of the energy $E$ for a mesoscopic ring with $N=8$ and $\phi=0.5$ in the limit of strong-coupling. (a) $V_{a}=V_{b}=0$, (b) $V_{a}=2$ and $V_{b}=0$, (c) $V_{a}=0$ and $V_{b}=2$ and (d) $V_{a}=V_{b}=2$.

for all energies. This reveals that the electron cannot conduct through the ring. Similar response is also observed when both the two inputs are high i.e., $V_{a}=V_{b}=2$, and in this case also the ring does not allow to pass an electron from the source to the drain (Fig. 2(d)). On the other hand, for the cases where any one of the two inputs is high and other is low i.e., either $V_{a}=2$ and $V_{b}=0$ (Fig. 2(b)) or $V_{a}=0$ and $V_{b}=2$ (Fig. 2(c)), the conductance exhibits fine resonant peaks for some particular energies. Thus for both these two cases the 
electron conduction takes place across the ring. At the resonances where the conductance approaches the value 2 , the transmission probability $T$ goes to unity, since the relation $g=2 T$ follows from the Landauer conductance formula (see Eq. 1 with $e=h=1$ ). All these resonant peaks are associated with the energy eigenvalues of the ring, and accordingly, we can say that the conductance spectrum manifests itself the electronic structure of the
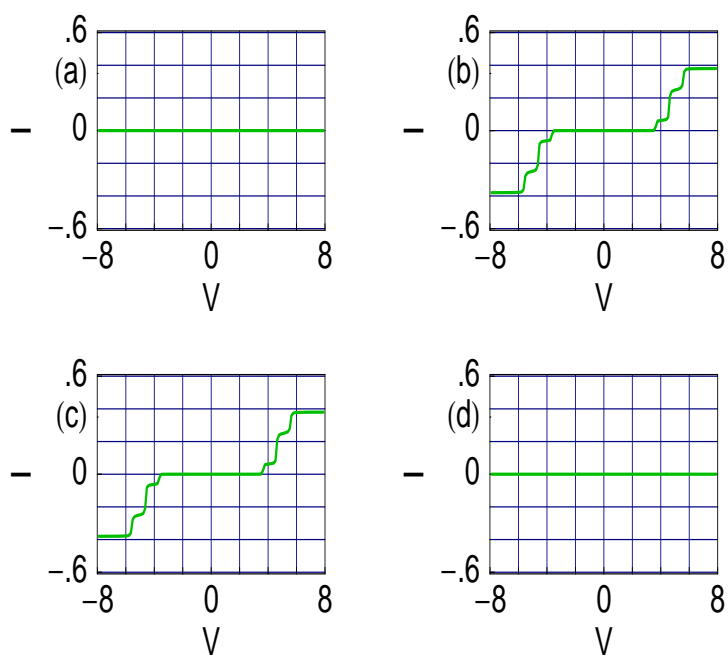

Figure 4: (Color online). Current $I$ as a function of the bias voltage $V$ for a mesoscopic ring with $N=8$ and $\phi=0.5$ in the limit of weak-coupling. (a) $V_{a}=V_{b}=0$, (b) $V_{a}=2$ and $V_{b}=0$, (c) $V_{a}=0$ and $V_{b}=2$ and (d) $V_{a}=V_{b}=2$.

ring. Thus more resonant peaks can be obtained for larger rings corresponding to their energy eigenvalues. Now we justify the dependences of the gate voltages on the electron transport for these four different cases. The probability amplitude of getting an electron across the ring depends on the quantum interference of the electronic waves passing through the upper and lower arms of the ring. For the symmetrically connected ring i.e., when the two arms of the ring are identical with each other, the probability amplitude is exactly zero $(T=0)$ for the flux $\phi=\phi_{0} / 2$. This is due to the result of the quantum interference among the two waves in the two arms of the ring, which can be obtained in a very simple mathematical calculation. Thus for the cases when both the two inputs $\left(V_{a}\right.$ and $\left.V_{b}\right)$ are either low or high, the transmission probability drops to zero. On the other hand, for the other two cases the symmetry of the two arms of the ring is broken by applying the gate voltage either in the atom $a$ or $b$, and therefore, the non-zero value of the transmission probability is achieved which reveals the electron conduction across the ring. Thus we can predict that the electron conduction takes place across the ring if one, and only one, of the inputs to the gate is high, while if both the inputs are low or

Table 1: XOR gate behavior in the limit of weakcoupling. The current $I$ is computed at the bias voltage 6.02 .

\begin{tabular}{|c|c|c|}
\hline \hline Input-I $\left(V_{a}\right)$ & Input-II $\left(V_{b}\right)$ & Current $(I)$ \\
\hline 0 & 0 & 0 \\
\hline 2 & 0 & 0.378 \\
\hline 0 & 2 & 0.378 \\
\hline 2 & 2 & 0 \\
\hline \hline
\end{tabular}

both are high the conduction is no longer possible. This feature clearly demonstrates the XOR behavior. With these characteristics, we get additional one feature when the coupling strength of the ring to the electrodes increases from the low regime to high one. In the limit of strong ring-to-electrodes coupling, all these resonances get substantial widths compared to the weak-coupling limit. The results are shown in Fig. 3, where all the other parameters are identical to those in Fig. 2. The contribution for the broadening of the resonant peaks in this strong-coupling limit appears from the imaginary parts of the self-energies $\Sigma_{S}$ and $\Sigma_{D}$, respectively 20 . Hence by tuning the coupling strength, we can get the electron transmission across the ring for the wider range of energies and it provides an important signature in the study of current-voltage $(I-V)$ characteristics.

All these features of electron transfer become much more clearly visible by studying the $I-V$ characteristics. The current passing through the ring is computed from the integration procedure of the transmission function $T$ as prescribed in Eq. 6. The transmission function varies exactly similar to that of the conductance spectrum, differ only in magnitude by the factor 2 since the relation $g=2 T$ holds from the Landauer conductance formula Eq. 1. As illustrative examples, in Fig. 4 we show the currentvoltage characteristics for a mesoscopic ring with $N=8$ in the limit of weak-coupling. For the cases when both the two inputs are identical with each other, either low (Fig. 4(a)) or high (Fig. 4(d)), the current is zero for the entire bias voltages. This behavior is clearly understood from the conductance 
spectra, Figs. 2(a) and (d), since the current is computed from the integration procedure of the transmission function $T$. For the two other cases where only one of the two inputs is high and other is low, a high output current is obtained which are clearly described in Figs. 4(b) and (c). From these figures it is observed that the current exhibits staircase-like structure with fine steps as a function of the applied bias voltage. This is due to the existence of the sharp resonant peaks in the conductance spectrum in the weak-coupling limit, since the current is computed by the integration method of the transmission
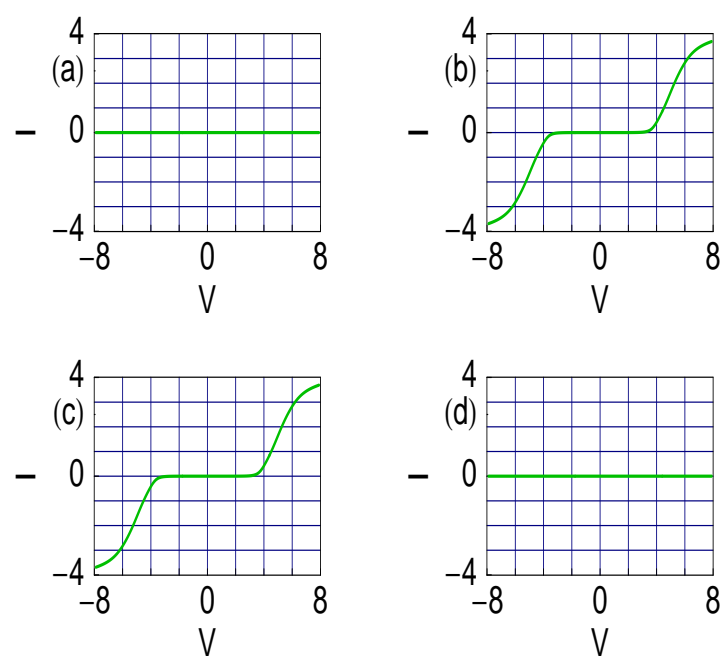

Figure 5: (Color online). Current $I$ as a function of the bias voltage $V$ for a mesoscopic ring with $N=8$ and $\phi=0.5$ in the limit of strong-coupling. (a) $V_{a}=V_{b}=0$, (b) $V_{a}=2$ and $V_{b}=0$, (c) $V_{a}=0$ and $V_{b}=2$ and (d) $V_{a}=V_{b}=2$.

function $T$. With the increase of the bias voltage $V$, the electrochemical potentials on the electrodes are shifted gradually, and finally cross one of the quantized energy levels of the ring. Accordingly, a current channel is opened up which provides a jump in the $I-V$ characteristic curve. Addition to these behaviors, it is also important to note that the non-zero value of the current appears beyond a finite value of $V$, so-called the threshold voltage $\left(V_{t h}\right)$. This $V_{t h}$ can be controlled by tuning the size $(N)$ of the ring. From these $I-V$ characteristics the behavior of the XOR gate response is clearly visible. To make it much clear, in Table 1. we present a quantitative estimate of the typical current amplitude, computed at the bias voltage $V=6.02$, in this weak-coupling limit. It shows that $I=0.378$ only when any one of the two inputs is high and other is low, while for the other cases when either $V_{a}=V_{b}=0$ or $V_{a}=V_{b}=2, I$ gets the value 0 . In the same analogy, as above, here we also discuss the $I-V$ characteristics for the strong-coupling limit. In this limit, the current varies almost continuously with the applied bias voltage and achieves much larger amplitude than the weak-coupling case as presented in Fig. 5] The reason is that, in the limit of strong-coupling all the energy levels get broadened which provide larger current in the integration procedure of the transmission function $T$. Thus by tuning the strength of the ring-to-electrodes coupling, we can achieve very large current amplitude

Table 2: XOR gate behavior in the limit of strongcoupling. The current $I$ is computed at the bias voltage 6.02 .

\begin{tabular}{|c|c|c|}
\hline \hline Input-I $\left(V_{a}\right)$ & Input-II $\left(V_{b}\right)$ & Current $(I)$ \\
\hline 0 & 0 & 0 \\
\hline 2 & 0 & 2.846 \\
\hline 0 & 2 & 2.846 \\
\hline 2 & 2 & 0 \\
\hline \hline
\end{tabular}

from the very low one for the same bias voltage $V$. All the other properties i.e., the dependences of the gate voltages on the $I-V$ characteristics are exactly similar to those as given in Fig. 4. In this strongcoupling limit we also make a quantitative study for the typical current amplitude, given in Table 2. where the current amplitude is determined at the same bias voltage $(V=6.02)$ as earlier. The response of the output current is exactly similar to that as given in Table 1 . Here the non-zero value of the current gets the value 2.846 which is much larger compared to the weak-coupling case which shows the value 0.378 . From these results we can clearly manifest that a mesoscopic ring exhibits the XOR gate response.

\section{Concluding remarks}

To summarize, we have addressed XOR gate response in a mesoscopic metallic ring threaded by a magnetic flux $\phi$. The ring, composed of identical quantum dots, is attached symmetrically to the source and drain. The upper and lower arms of the ring are subjected to the gate voltages $V_{a}$ and $V_{b}$, respectively those are taken as the two inputs of the XOR gate. A simple tight-binding model is used to describe the full system and all the calcu- 
lations are done in the Green's function formalism. We have numerically computed the conductanceenergy and current-voltage characteristics as functions of the ring-electrodes coupling strengths, magnetic flux and gate voltages. Very interestingly we have noticed that, for the half flux-quantum value of $\phi\left(\phi=\phi_{0} / 2\right)$, a high output current (1) (in the logical sense) appears if one, and only one, of the inputs to the gate is high (1). On the other hand, if both the inputs are low (0) or both are high (1), a low output current (0) appears. It clearly manifests the XOR gate behavior and this aspect may be utilized in designing a tailor made electronic logic gate. In view of the potential application of this XOR gate as a circuit element in an integrated circuit, we would like to point out that care should be taken during the application of the magnetic field in the ring such that the other circuit elements of the integrated circuit are not effected by this field.

In this presentation, we have calculated all the results by ignoring the effects of the temperature, electron-electron correlation, disorder, etc. Due to these factors, any scattering process that appears in the arms of the ring would have influence on electronic phases, and, in consequences can disturb the quantum interference effects. Here we have assumed that, in our sample all these effects are too small, and accordingly, we have neglected all these effects in our present study.

The importance of this article is mainly concerned with (i) the simplicity of the geometry and (ii) the smallness of the size. To the best of our knowledge the XOR gate response in such a simple low-dimensional system has not been addressed earlier in the literature.

\section{References}

[1] A. Aviram, M. Ratner, Chem. Phys. Lett. 29 (1974) 277.

[2] T. Dadosh, Y. Gordin, R. Krahne, I. Khivrich, D. Mahalu, V. Frydman, J. Sperling, A. Yacoby, I. Bar-Joseph, Nature 436 (2005) 677.

[3] J. Chen, M. A. Reed, A. M. Rawlett, J. M. Tour, Science 286 (1999) 1550.

[4] M. A. Reed, C. Zhou, C. J. Muller, T. P. Burgin, J. M. Tour, Science 278 (1997) 252.

[5] P. A. Orellana, M. L. Ladron de Guevara, M. Pacheco, A. Latge, Phys. Rev. B 68 (2003) 195321.
[6] P. A. Orellana, F. Dominguez-Adame, I. Gomez, M. L. Ladron de Guevara, Phys. Rev. B 67 (2003) 085321.

[7] A. Nitzan, Annu. Rev. Phys. Chem. 52 (2001) 681.

[8] A. Nitzan, M. A. Ratner, Science 300 (2003) 1384.

[9] D. M. Newns, Phys. Rev. 178 (1969) 1123.

[10] V. Mujica, M. Kemp, M. A. Ratner, J. Chem. Phys. 101 (1994) 6849.

[11] V. Mujica, M. Kemp, A. E. Roitberg, M. A. Ratner, J. Chem. Phys. 104 (1996) 7296.

[12] K. Walczak, Phys. Stat. Sol. (b) 241 (2004) 2555 .

[13] K. Walczak, arXiv:0309666.

[14] W. Y. Cui, S. Z. Wu, G. Jin, X. Zhao, Y. Q. Ma, Eur. Phys. J. B. 59 (2007) 47.

[15] R. Baer, D. Neuhauser, J. Am. Chem. Soc. 124 (2002) 4200.

[16] D. Walter, D. Neuhauser, R. Baer, Chem. Phys. 299 (2004) 139.

[17] K. Tagami, L. Wang, M. Tsukada, Nano Lett. 4 (2004) 209.

[18] K. Walczak, Cent. Eur. J. Chem. 2 (2004) 524.

[19] R. Baer, D. Neuhauser, Chem. Phys. 281 (2002) 353.

[20] S. Datta, Electronic transport in mesoscopic systems, Cambridge University Press, Cambridge (1997).

[21] M. B. Nardelli, Phys. Rev. B 60 (1999) 7828. 